\title{
A CHARACTERIZATION OF ACYCLIC SWITCHING CLASSES OF GRAPHS USING FORBIDDEN SUBGRAPHS
}

\author{
JURRIAAN HAGE* AND TERO HARJU ${ }^{\dagger}$
}

\begin{abstract}
We characterize the switching classes that do not contain an acyclic graph. The characterization is by means of a set of forbidden graphs. We prove that in addition to switches of the cycles $C_{n}$ for $n \geq 7$ there are only finitely many such graphs in 24 switching classes, all having at most 9 vertices. We give a representative of each of the 24 switching classes.
\end{abstract}

Key words. Graphs, switching class. Seidel switching, acyclic graphs, trees, forbidden graphs, critically cyclic graphs

AMS subject classifications. 05

1. Introduction. Blue things are new, red things are replaced (by the blue if applicable)

For a finite undirected graph $G=(V, E)$ and a set $\sigma \subseteq V$, the switch of $G$ by $\sigma$ is defined as the graph $G^{\sigma}=\left(V, E^{\prime}\right)$, which is obtained from $G$ by removing all edges between $\sigma$ and its complement $\bar{\sigma}$ and adding as edges all nonedges between $\sigma$ and $\bar{\sigma}$. The switching class $[G]$ determined by $G$ consists of all switches $G^{\sigma}$ for subsets $\sigma \subseteq V$

A switching class is an equivalence class of graphs under switching. The initiators of the theory of switching classes of graphs were Van Lint and Seidel [7]. They used the model in their investigation of elliptic geometry. For a survey of switching classes of graphs, and especially their many connections to other parts of mathematics, we refer to Seidel [8], Seidel and Taylor [9], and Cameron [2]. Recently a book by Ehrenfeucht, Harju and Rozenberg was published on 2-structures that has a number of chapters on switching classes of graphs and their generalizations [4]. A book completely devoted to the subject is the first author's thesis (Hage [5]). Part of the motivation for the general model treated in the latter two books is that they constitute a way in which to model the semantics of a certain type of networks of processors.

In this paper we solve a problem raised by Acharya [1] and mentioned by Zaslavsky in his dynamic survey in 1999 [11], which asks for a characterization of those graphs that have an acyclic switch. We are concerned with those graphs that do not have an acyclic switch. Such graphs will be called forbidden. Obviously, if a forbidden graph occurs in another graph, then the latter is also forbidden. For this reason we are interested in the graphs that are minimal in this respect: they do not have an acyclic switch, but all their induced subgraphs do have an acyclic switch. We call these graphs and the corresponding switching class critically cyclic. We show that apart from the simple cycles $C_{n}$ for $n \geq 7$, there are only finitely many critically cyclic graphs. In fact, we shall prove that a critically cyclic graph $G \notin\left[C_{n}\right]$ has order at most 9. These graphs are partitioned into 24 switching classes, and altogether there are 905 critically cyclic graphs (up to isomorphism and excluding switches of the cycles $\left.C_{n}\right){ }^{3}$

\footnotetext{
${ }^{*}$ Corresponding author - Institute of Information and Computing Science, University of Utrecht, P.O.Box 80.089, 3508 TB Utrecht, Netherlands; email jur@cs.uu.nl

${ }^{\dagger}$ Department of Mathematics, University of Turku, FIN-20014 Turku, Finland; email harju@utu.fi

${ }^{3}$ Contact the first author for the postscript-file containing all of them.
} 
In order to save the reader from long - and occasionally tedious - technical constructions for the small graphs, we rely on a computer program (in fact, two independent ones) for the cases of order at most 9. Therefore our purpose is to prove that if $G$ is a critically cyclic graph of order $n \geq 10$, then $G \in\left[C_{n}\right]$. The proof of this result uses the characterization from [6] of the acyclic graphs $G$ - henceforth called the special acyclic graphs - that have a non-trivial acyclic switch (see Section 4).

The paper is structured as follows. After some preliminaries we list the necessary details of the special acyclic graphs from [6]. We proceed by proving that critically cyclic graphs can have only a limited number of isolated vertices. As a consequence, a vertex in a critically cyclic graph has only a limited number of leaves adjacent to it. We prove that each switching class consisting of critically cyclic graphs and different from $\left[C_{n}\right]$ for $n \geq 8$, contains a graph $G$ that is almost a special acyclic graph. We then prove by case analysis relying on the types of the special acyclic graphs that a critically cyclic graph must have order at most 9 . At the end of the paper we shall spend some time discussing the computer programs that were used to search for the small critically cyclic graphs. We shall also consider the question of why not all of the critically cyclic switching classes are used in our proof.

2. Preliminaries. For a (finite) set $V$, let $|V|$ be the cardinality of $V$. We shall often identify a subset $A \subseteq V$ with its characteristic function $A: V \rightarrow \mathbf{Z}_{2}$, where $\mathbf{Z}_{2}=\{0,1\}$ is the cyclic group of order two, by the convention that for $x \in V$, $A(x)=1$ if and only if $x \in A$. The symmetric difference of two sets $A$ and $B$ is denoted by $A+B$, and for the difference between $A$ and $B$ we write $A-B$.

The set $E(V)=\{\{x, y\} \mid x, y \in V, x \neq y\}$ is the set of all unordered pairs of distinct elements of $V$. A graph is a pair $G=(V, E)$ where $V$ is the set of vertices and $E \subseteq E(V)$ the set of edges. We write $x y$ or $y x$ for the undirected edge $\{x, y\} \in E$; we call $x$ and $y$ adjacent. The graphs of this paper will be finite, undirected and simple, i.e., they contain no loops or multiple edges. The cardinalities $|V|$ and $|E|$ are called the order and the size of $G$. Analogously to sets, a graph $G$ will be identified with the characteristic function $G: E(V) \rightarrow \mathbf{Z}_{2}$ of its set of edges defined by $G(x y)=1$ for $x y \in E$ and $G(x y)=0$ for $x y \notin E$. Later we shall use both notations, $G=(V, E)$ and $G: E(V) \rightarrow \mathbf{Z}_{2}$, for graphs.

A graph $H=\left(X, E^{\prime}\right)$ is a subgraph of $G=(V, E)$, if $X \subseteq V$ and $E^{\prime} \subseteq E$. Moreover, if $H \neq G$ then $H$ is a proper subgraph of $G$. Also, $H$ is an induced subgraph or a subgraph induced by $X$ if for all distinct vertices $x, y \in X, H(x y)=G(x y)$. As shorthand we write $G-x$ for the subgraph induced by $V-\{x\}$ and, more generally, we write $G-I$ for the subgraph induced by $V-I$. Let $H$ be a subgraph induced by a nonempty set $X \subseteq V$. If $H(x y)=1$ for all distinct $x, y \in X$, then $H$ is called a clique. On the other hand, if $H(x y)=0$ for all distinct $x, y \in X$, then $X$ is said to be independent.

For two graphs $G$ and $H$ on the vertex set $V$, we define $G+H$ to be the graph such that $(G+H)(x y)=G(x y)+H(x y)$ for all $x y \in E(V)$, where + is addition modulo 2. We extend this operation to graphs on different sets of vertices $V$ and $V^{\prime}$ respectively, by first extending them to graphs on $V \cup V^{\prime}$ and setting all new edges to 0 .

The disjoint union of two graphs $G$ and $H$ on the other hand is denoted $G \cup H$. We use $k \cdot G$ as shorthand for the disjoint union of $k$ copies of $G$.

Some graphs we shall encounter in the sequel are $K_{n}$, the clique on $n$ vertices, and $K_{m, n}$, the complete bipartite graph on two disjoint sets of $m$ and $n$ vertices respectively. $P_{n}$ denotes a path of $n$ vertices and $C_{n}$ denotes a cycle on $n$ vertices. 
For a vertex $v \in V$ of a graph $G$, the set $N_{G}(v) \subseteq V$ is the set of vertices adjacent to $v$ in $G$. The degree of $v$ is defined by $d_{G}(v)=\left|N_{G}(v)\right|$. An isolated vertex has degree zero, a leaf degree one. A vertex $v$ is a leaf at $z$ if $v$ is a leaf adjacent to $z$.

A graph is acyclic if it has no cycles. A tree is a connected acyclic graph.

A selector for $G=(V, E)$ is a subset $\sigma \subseteq V$, or alternatively a function $\sigma: G \rightarrow \mathbf{Z}_{2}$. A switch of a graph $G$ by $\sigma$ is the graph $G^{\sigma}$ such that for all $x y \in E(V)$,

$$
G^{\sigma}(x y)=\sigma(x)+G(x y)+\sigma(y) .
$$

It should be clear that this definition of switching is equivalent to the one given in the introduction. One of the switches of the graph (7-3) of Figure 3.1 is the graph (7-3') of Figure 3.4. In the figures we shall usually indicate a selector by the black vertices.

For a singleton set $\sigma=\{x\}$ we shall write $G^{x}$ instead of $G^{\{x\}}$.

The set $[G]=\left\{G^{\sigma} \mid \sigma \subseteq V\right\}$ is called the switching class of $G=(V, E)$. We reserve lower case $\sigma, \tau$ for selectors (subsets) used in switching.

We always have $G^{\sigma}=G^{\bar{\sigma}}$ for the complemented selector $\bar{\sigma}=V-\sigma$.

A selector $\sigma$ is said to be constant on a subset $X \subseteq V$ if $\sigma$ is a constant function on $X$, that is, if $X \subseteq \sigma$ or $X \cap \sigma=\emptyset$. Note that if $\sigma$ is constant on $X$, then the subgraphs induced by $X$ in $G$ and in $G^{\sigma}$ are equal.

3. Critically cyclic graphs. A graph $G$ is said to be forbidden if $G$ does not have an acyclic switch. Moreover, a forbidden graph $G$, as well as its switching class $[G]$, is called critically cyclic if all proper induced subgraphs of $G$ have an acyclic switch. It is clear that a switch of a critically cyclic graphs is also critically cyclic.

We say that an acyclic graph $G$ has a singular acyclic switch, if $G$ has a unique acyclic switch different from $G$, that is, if $\sigma$ and $\tau$ are any two nonconstant selectors for which both $G^{\sigma}$ and $G^{\tau}$ are acyclic, then $G^{\sigma}$ and $G^{\tau}$ are equal (not only isomorphic).

Let $G$ be a critically cyclic graph. By definition, for all $x \in V$, there is a switch $G^{\sigma}$ such that $G^{\sigma}-x$ is acyclic. We have the following simple result that will be used quite often without reference in the proofs.

Lemma 3.1. Let $G$ be a critically cyclic graph and let $x$ be a vertex of $G$.

(i) The proper subgraphs of $G$ all have acyclic switches.

(ii) There exists a switch $G^{\sigma}$ of $G$ such that the subgraph $G^{\sigma}-x$ is acyclic. In this case all cycles of $G^{\sigma}$ and of $\left(G^{x}\right)^{\sigma}$ go through $x$.

Note that it is not true that in every critically cyclic graph $G$ there is a vertex $x$ such that $G-x$ is acyclic; the graph $K_{3,3} \cup 3 \cdot K_{1}$ of Figure 3.3(9-2) is a counter example.

EXAMPLE 3.2. Let $G$ be the graph (7-3') in Figure 3.4. We prove that it is a critically cyclic graph. For this we need to show that it has no acyclic switches and removing any of the vertices allows for an acyclic switch. For the latter it is sufficient to observe that the vertices $2, \ldots, 6$ are all on the unique cycle of $G$, and the subgraphs $G^{\{2,5\}}-7$ and $G^{\{3,6\}}-1$ are acyclic.

To prove that $G$ has no acyclic switch observe that $G$ has seven edges and an acyclic graph can have at most six. We prove that applying any selector will not decrease the number of edges, and thereby we have proved that there is no acyclic switch of $G$. Since $G^{\sigma}=G^{\bar{\sigma}}$ for all selectors $\sigma$, we can assume that $\sigma$ has at most three vertices.

First of all, for all vertices $x, d_{G}(x) \leq 3=(n-1) / 2$. Hence applying a singleton selector cannot decrease the number of edges.

For doubleton selectors, $\sigma=\left\{x_{1}, x_{2}\right\}$, we can reason in the same way. The number of edges that change is $|\sigma| \cdot(7-|\sigma|)=10$. We must make sure that every 
selector makes at most five edges disappear. The only possible way, knowing that the maximum degree is three, is to take $\sigma=\{2,6\}$, but in that case only four edges are removed, because one edge occurs inside the subgraph induced by $\sigma$.

For selectors of size 3 , twelve edges will change. Hence we must look for selectors $\sigma$ which create fewer than six edges, that is, $\sigma$ make more than six edges of $G$ disappear. For this, the selector must contain a vertex of degree three, say $\{2\}$. If also $6 \in \sigma$, then the number of edges to be removed is four. Since there are no other vertices of degree three in $G$, we conclude that $6 \notin \sigma$. If $\sigma$ has two vertices of degree two, then the subset $\sigma$ has at most six edges going to its complement, because either the two of them are adjacent, or one of them is adjacent to 2 .

Note that $C_{n}$ for $n \leq 6$ has an acyclic switch: take an independent set of cardinality $\lfloor n / 2\rfloor$. However, the following was already proved by Acharya [1].

Lemma 3.3. The cycle $C_{n}$ is critically cyclic for each $n \geq 7$.

Proof. Let $G=C_{n}$ with $n \geq 7$. First of all, removing any vertex from $G=C_{n}$ gives us an acyclic graph $P_{n-1}$. Hence we need to prove only that all switches of $G$ have a cycle. Let $\left\{x_{1}, x_{2}, \ldots, x_{n}\right\}$ be the vertices of $G$ where $G\left(x_{i} x_{i+1}\right)=1=G\left(x_{n} x_{1}\right)$. Suppose that $G^{\sigma}$ is acyclic.

Assume first that $\sigma$ has the same value for two adjacent vertices, say $\sigma\left(x_{1}\right)=1=$ $\sigma\left(x_{2}\right)$. Now $\sigma\left(x_{i}\right)=1$ for each $4 \leq i \leq n-1$, since $\left\{x_{1}, x_{2}, x_{i}\right\}$ does not induce a triangle $C_{3}$ in $G^{\sigma}$. Also $\sigma\left(x_{3}\right)=1$ and $\sigma\left(x_{n}\right)=1$ because otherwise $\left\{x_{3}, x_{n-1}, x_{n-2}\right\}$ and $\left\{x_{n}, x_{4}, x_{5}\right\}$ induce a triangle in $G^{\sigma}$. However, now $\sigma$ is constant and $G^{\sigma}=G$; contradiction. This takes care of all $C_{n}$ where $n \geq 7$ is odd.

There remains the case where $\sigma$ contains every other vertex of $G$. In this case, it is easy to see that if $n=8$, then $G^{\sigma}$ is again a cycle $C_{n}$, and if $n \geq 10$ and $n$ is even, then the subset $\left\{x_{1}, x_{2}, x_{4}, x_{5}, x_{7}, x_{8}\right\}$ induces a cycle $C_{6}$ in $C_{n}^{\sigma}$. These contradictions prove the claim.

We now state the result of our computer search for critically cyclic graphs.

THEOREM 3.4. There are 27 switching classes of critically cyclic graphs of order $n \leq 9$. Representatives of these switching classes are given in Figures 3.1, 3.2 and 3.3.

The main theorem proved in this paper is the following.

THEOREM 3.5. The switching classes $\left[C_{n}\right]$ are the only critically cyclic switching classes of order $n \geq 10$.

In the following proofs we shall refer to the graphs from Figures $3.1-3.4$. The black vertices in Figure 3.4 indicate how these graphs can be switched into the corresponding graphs from the former three figures.

4. The special acyclic graphs. We shall now describe the special acyclic graphs of [6] (see Figure 4.1) that will be often referred to in the rest of the paper. These acyclic graphs $S$ have the property that they have an acyclic switch $S^{\sigma}$ different from $S$.

Type (1s). The graph in Figure $4.1(1 \mathrm{~s})$ is denoted by $S_{k, m, l}$. It is obtained from the graph $K_{1, k+m}$ by substituting $k$ leaves by an edge and by adding $l$ isolated vertices. To be precise, $S=S_{k, m, l}$ consists of the induced subgraphs $H, I$ and $M$ as defined in $(\mathrm{S} 1)-(\mathrm{S} 3)$.

(S1) $H=\{z\} \cup\left\{y_{i}, x_{i} \mid i=1,2, \ldots, k\right\}$ consists of vertices for which $G\left(z y_{i}\right)=1=$ $G\left(y_{i} x_{i}\right)$ for each $i$. The vertex $z$ is called the centre of $S$.

(S2) $I=\left\{u_{1}, u_{2}, \ldots, u_{l}\right\}$ consists of isolated vertices.

(S3) $M=\left\{v_{1}, v_{2}, \ldots, v_{m}\right\}$ consists of leaves such that $G\left(z v_{i}\right)=1$ for each $i$. 


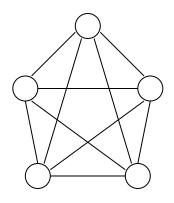

$(5-1)$

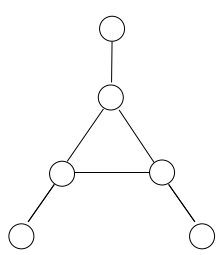

(6-1)

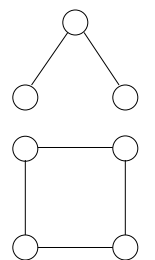

(7-1)

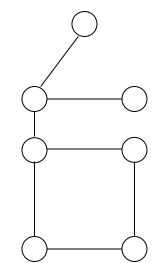

$(7-2)$

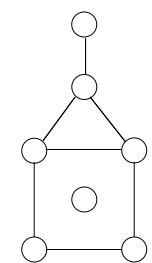

(7-3)<smiles>OC1OOO1</smiles>

$(7-4)$

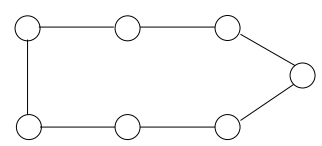

$(7-5)$

FIG. 3.1. The critically cyclic graphs of order 5, 6 and 7<smiles></smiles>

$(8-1)$<smiles>OOC1OOO1</smiles>

$(8-4)$

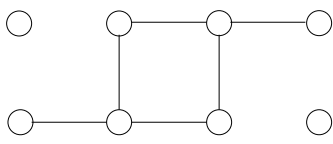

$(8-8)$

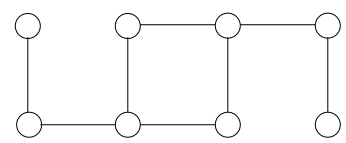

$(8-2)$

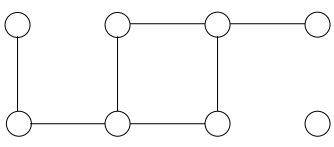

$(8-3)$

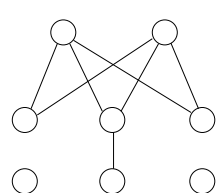

$(8-5)$

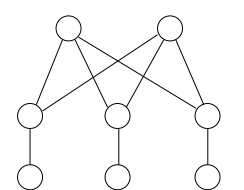

(8-6)

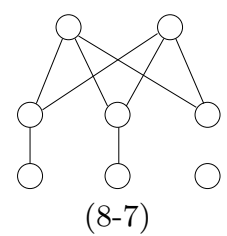

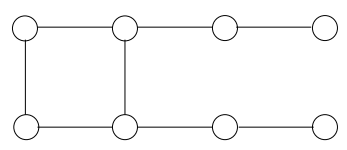

(8-9)

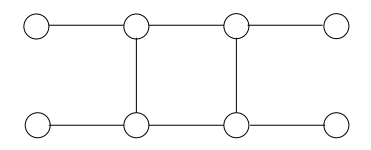

$(8-10)$

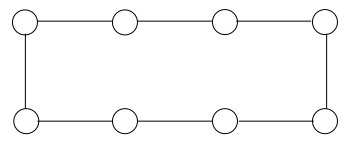

$(8-11)$<smiles>OOOOOO</smiles>

$(8-12)$

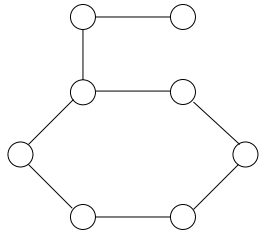

$(8-13)$

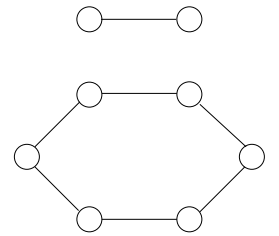

$(8-14)$

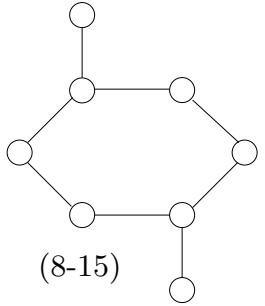

FIG. 3.2. Critically cyclic graphs of order 8 


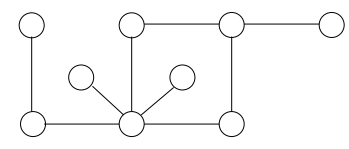

$(9-1)$

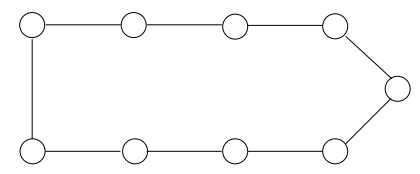

(9-4)

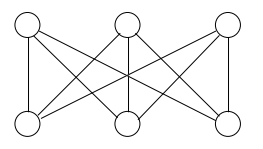

(9-2)

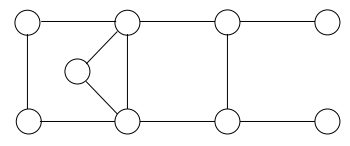

(9-3)

FIG. 3.3. Critically cyclic graphs of order 9

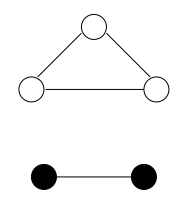

$\left(5-1^{\prime}\right)$

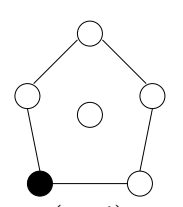

(6-1')

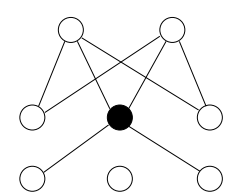

(8-5')

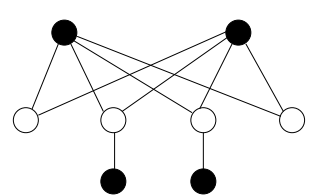

$\left(8-8^{\prime}\right)$

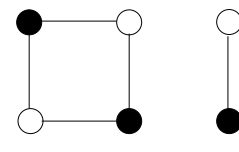

$\left(7-2^{\prime}\right)$

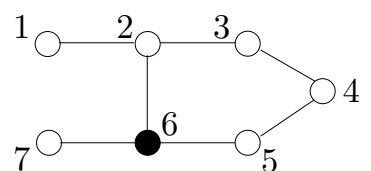

(7-3')

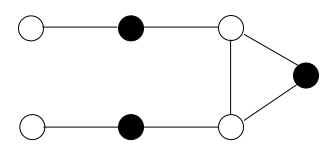

$\left(7-5^{\prime}\right)$

FIG. 3.4. Switches of known critically cyclic graphs that are used in the proofs

For the types (As) with $A=2,3, \ldots 8$ the corresponding special acyclic graphs will be denoted by $S_{A}(k, m)$, where $k$ and $m$ indicate the number of leaves of the (black) vertices $z_{1}$ and $z_{2}$. Because of the symmetry in $k$ and $m$ in each of these graphs we may assume that $k \geq m$.

Denote by $P_{t}(m, k)$ the tree that is obtained from the path $P_{t}$ of $t$ vertices when the leaves are substituted by $K_{1, m}$ and $K_{1, k}$ (see Figure 4.1(4s) for $P_{3}(k, m)$ ).

Type (2s). $S_{2}(k, m)=K_{1, k} \cup K_{1, m}$.

Type (3s). $S_{3}(k, m)=K_{1, k} \cup K_{1, m} \cup K_{1}$.

Type (4s). $S_{4}(k, m)=P_{3}(k, m)$.

Type (5s). $S_{5}(k, m)=P_{3}(k, m) \cup K_{1}$.

Type (6s). $S_{6}(k, m)=P_{2}(k, m)$.

Type (7s).. $\quad S_{7}(k, m)$ is equal to $K_{1,3}(k, m)$ where two leaves of $K_{1,3}$ are substituted by $K_{1, k}$ and $K_{1, m}$ (see Figure $4.1(7 \mathrm{~s})$ ).

Type (8s). $S_{8}(k, m)=P_{4}(k, m)$.

Types $(9 \mathrm{~s})-(\mathbf{1 2 s})$. The acyclic graphs of these types are $P_{7}, T_{7}, P_{6}$, and $P_{4} \cup P_{2}$. These are listed in Figures $4.1(9 \mathrm{~s})-(12 \mathrm{~s})$.

A small acyclic graph can be of several of the above types. The role of the small acyclic graphs of the types $(9 \mathrm{~s})-(12 \mathrm{~s})$ is strictly limited in this paper, because of their low order. Notice that $P_{6}$ equals $P_{4}(1,1)$ of the type $(8 \mathrm{~s})$, but we treat this small instance independently.

In $[6]$ we proved the following theorem. 


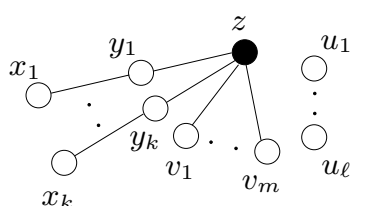

$(1 \mathrm{~s})$

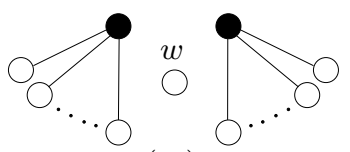

(3s)
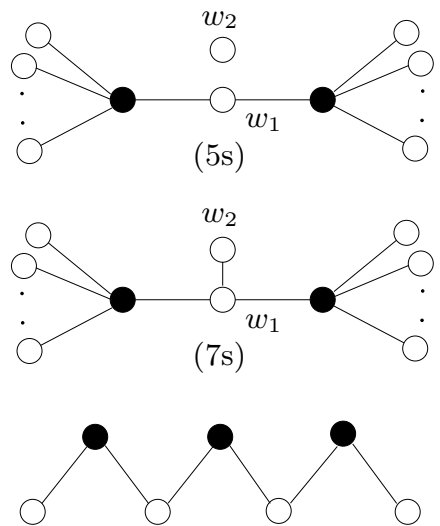

$(9 \mathrm{~s})$

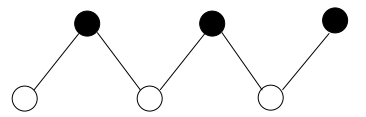

$(11 \mathrm{~s})$

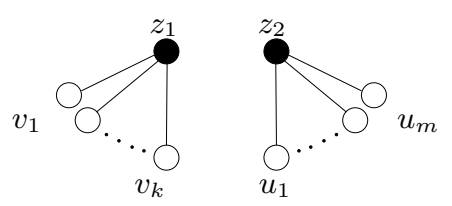

$(2 \mathrm{~s})$

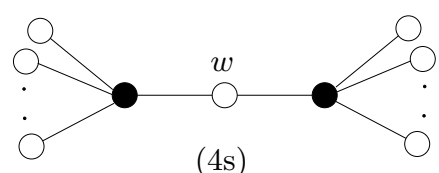

$(4 \mathrm{~s})$
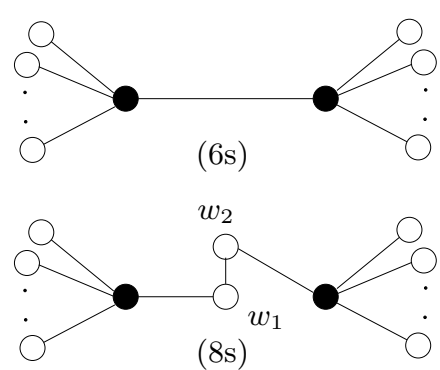

$(8 \mathrm{~s})$

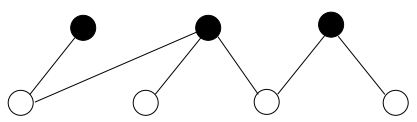

(10s)

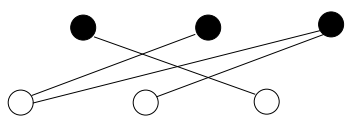

$(12 \mathrm{~s})$

FIG. 4.1. The special acyclic graphs (1s)-(12s)

THEOREM 4.1. Every switching class contains at most three acyclic graphs up to isomorphism. The acyclic graphs $G$ that have an acyclic switch $G^{\sigma}$ by a nonconstant selector $\sigma$ are the special graphs of the types (1s) - (12s).

The blackened vertices in Figure 4.1 constitute the centres of the special acyclic graphs $S$. We denote by $Z(S)$ the set of these centres. Notice that $Z(S)$ consists of either one or two vertices.

Let $\sigma=Z(S)$ for a special acyclic graph $S$. We observe that $S^{\sigma}$ is of the same type as $S$ except for a few of the cases: A graph of the type (3s) switches into a graph of the type (4s) (and vice versa). The graphs (11s) and (12s) switch into each other.

We shall often want to use the fact that a certain special acyclic graph $S$ has a singular acyclic switch, that is, a unique acyclic switch by a nonconstant selector. We shall now give a list of small special acyclic graphs that do have a singular acyclic switch. We omit the proof of Lemma 4.2, because the graphs in it are small and the claim can be easily checked even by hand although some work is required.

LEMMA 4.2. The following special acyclic graphs $S$ have a singular acyclic switch:

(i) $S_{1,2,2}$ and $S_{0,3,3}$ of the type (1s), 
(ii) $S_{A}(2,2)$ of the types (As) for $A=2,3,4$,

(iii) $S_{A}(2,1)$ of the types (As) for $A=5,7,8$, and

(iv) $S_{6}(3,3)$ of the type (6s).

In these cases the singular acyclic switch $S^{\sigma}$ is obtained by the selector $\sigma=Z(S)$ and its complement $\bar{\sigma}$.

We turn now to special acyclic graphs that are more general in their structure.

LEMma 4.3. The special acyclic graph $S=S_{A}(k, m)$ has a singular acyclic switch if

(i) $k, m \geq 2$ for the types (2s), (3s), and (4s),

(ii) $k \geq 2, m \geq 1$ for the types (5s), (7s), and (8s),

(iii) $k, m \geq 3$ for the type (6s).

In these cases the singular acyclic switch $S^{\sigma}$ is obtained by the selector $\sigma=Z(S)$ and its complement $\bar{\sigma}$.

Proof. In each of the cases under consideration the special acyclic graph $S=$ $S_{A}(k, m)$ has two centres, say $Z(S)=\left\{z_{1}, z_{2}\right\}$. Let $S^{\sigma} \neq S$ for a nonconstant selector $\sigma$ such that $S^{\sigma}$ is acyclic. We may assume that $\sigma\left(z_{1}\right)=1$. In each of these cases every pair $x, y \notin Z(S)$ of distinct vertices of $S$ belongs to an induced subgraph $R_{x, y}$ from Lemma 4.2 such that $Z(S) \subseteq R_{x, y}$ and $R_{x, y}$ is of the same type as $S$. When $\sigma$ is restricted to $R_{x, y}$, we have that the switch $R_{x, y}^{\sigma}$ is acyclic. By Lemma 4.2, $\sigma(x)=\sigma(y)$, from which the claim follows. $\square$

LEMma 4.4. The special acyclic graph $S=S_{k, m, l}$ has a singular acyclic switch in the following cases:

(i) $k \geq 3$,

(ii) $k=2$ and $m+l \geq 2$,

(iii) $k=1$ and $m, l \geq 2$, and

(iv) $k=0$ and $m, l \geq 3$.

In these cases the singular acyclic switch $S^{\sigma}$ is obtained by the singleton selector $\sigma=Z(S)$ and its complement $\bar{\sigma}$.

Proof. Let $S=S_{k, m, l}$ with $Z(S)=\{z\}$ be as described in (S1) - (S3) where $k, m$ and $l$ satisfy the requirements of the claim. Let $\sigma$ be a nonconstant selector such that $S^{\sigma}$ is acyclic. Since $S^{\sigma}=S^{\bar{\sigma}}$, we may assume that $\sigma(z)=1$. We shall show that $\sigma=\{z\}$ from which the claim follows. Note that the subgraph $S-z$ of $S$ equals the disjoint union $k \cdot P_{2} \cup(m+l) \cdot K_{1}$.

Suppose that $k \geq 3$. Now $S-z$ is not special and by Theorem 4.1, $\sigma$ must be constant on $S-z$. Therefore $\sigma=\{z\}$.

Suppose then that $k=2$ and $m+l \geq 2$. In this case, $S-z$ equals $2 \cdot P_{2} \cup 2 \cdot K_{1}$, which is not special. As in the above, we have $\sigma=\{z\}$.

For the rest of the cases where $k \leq 1$, the claim follows from Lemma 4.2 as in the proof of Lemma 4.3, since now every pair of vertices of $S$ belongs to an induced subgraph $S_{1,2,2}$ or $S_{0,3,3}$ that contains the centre $z$ of $S$.

5. Isolated vertices. In this section we give constraints for the isolated vertices in critically cyclic graphs. In particular, we prove our main tool for the final proof: if $G$ is critically cyclic and is such that $G-x$ is acyclic for a vertex $x$, then $G-x$ has no isolated vertices.

LEMma 5.1. A critically cyclic graph $G$ has at most two isolated vertices or $G=K_{3,3} \cup 3 \cdot K_{1}$ (see (9-2) in Figure 3.3).

Proof. Let $I=\left\{x_{1}, x_{2}, \ldots, x_{m}\right\}$ be the set of the isolated vertices in $G$. We assume that $m \geq 3$. The graph $G$ is critically cyclic, and hence $G-x_{1}$ is not acyclic 
but it has an acyclic switch $\left(G-x_{1}\right)^{\tau}$. The subgraph $G-I$ has a cycle, and therefore $\tau$ is not constant on $G-I$, say $\tau\left(v_{0}\right)=0$ and $\tau\left(v_{1}\right)=1$ for some $v_{0}, v_{1} \in V(G)-I$.

We show that $\tau$ has different values on the elements of $I-\left\{x_{1}\right\}$. From this it follows that $m=3$. For this purpose, suppose that there are two vertices, say $x_{2}$ and $x_{3}$, in $I-\left\{x_{1}\right\}$ having the same value. Without loss of generality we may assume that $\tau\left(x_{2}\right)=1=\tau\left(x_{3}\right)$.

If $\tau(v)=0$ for a vertex $v \in V-\left\{v_{0}\right\}$, then $\left(x_{2}, v_{0}, x_{3}, v\right)$ is a cycle in $\left(G-x_{1}\right)^{\tau}$, which contradicts the choice of $\tau$. However, if $\tau(x)=1$ for all $x \in I-\left\{x_{1}, x_{2}, x_{3}\right\}$, then $x_{1}$ is a leaf at $v_{0}$ in $G^{\tau}$, contradicting the fact that all cycles of $G^{\tau}$ go through $x_{1}$. This proves that the vertices of $I-\left\{x_{1}\right\}$ have different values in $\tau$. Hence $m=3$ with $\tau\left(x_{2}\right) \neq \tau\left(x_{3}\right)$.

Since $\left(G-x_{1}\right)^{\tau}$ is acyclic, and $G^{\tau}\left(x_{2} x_{3}\right)=1$, and every vertex of $V-I$ is adjacent to either $x_{2}$ or $x_{3}$, it follows that $V-I$ is independent in $\left(G-x_{1}\right)^{\tau}$. The switching class of a discrete graph of order $n$ consists of the complete bipartite graphs of order $n$ (see [8]), and therefore $G=K_{r, s} \cup 3 \cdot K_{1}$ where $r, s \geq 2$ since $G$ is not acyclic. Since $K_{3,3} \cup 3 \cdot K_{1}$ is a critically cyclic graph, and each $K_{2, s} \cup 3 \cdot K_{1}$, for $s \geq 4$, has an acyclic switch (by switching one of the vertices in the part of size 2 of $K_{2, s}$ ), the claim follows.

The following lemma is an immediate corollary to the previous result.

Lemma 5.2. Let $G$ be critically cyclic of order $n \geq 10$. Then no vertex $v \in V$ is adjacent to more than two leaves of $G$.

Proof. Let $L$ be a set of leaves of $G$ such that $G(u v)=1$ for all $u \in L$. Then the vertices of $L$ are isolated in $G^{\sigma}$ for the selector $\sigma=\{v\}$. By Lemma 5.1, $L$ has at most two elements, since the graph $K_{3,3} \cup 3 \cdot K_{1}$ is of order 9 .

In the proof of the following result we require knowledge of the small critically cyclic graphs. We shall say that a graph $G$ avoids (c.i) if $G$ does not contain the graph in Figure 3.1(c.i) for $c=5,6,7$ and Figure 3.2(c.i) for $c=8$, and Figure 3.3(c.i) for $c=9$ as an induced subgraph.

Recall that everry proper subgraph of a critically cyclic graph can be switched to a graph with no cycles. In particular, we have the following corollary that is used often in the rest of this paper.

Lemma 5.3. Let $G$ be a critically cyclic graph of order $n \geq 10$. Then $G$ avoids the graphs in Figures 3.1, 3.2 and 3.3.

The next lemma is our first general tool concerning isolated vertices.

Lemma 5.4. Let $G$ be a critically cyclic graph of order $n \geq 10$. Then $G$ has at most one isolated vertex.

Proof. By Lemma 5.1, $G$ has at most two isolated vertices. Suppose that $G$ has exactly two isolated vertices, say $I=\left\{x_{1}, x_{2}\right\}$. By assumption, $G$ is critically cyclic, and therefore there exists a selector $\tau$ such that $\left(G-x_{1}\right)^{\tau}$ is acyclic. We can assume without restriction that $\tau\left(x_{2}\right)=0$.

The set $\tau$ is independent in $G$, as well as in $\left(G-x_{1}\right)^{\tau}$, for otherwise, there is a triangle containing $x_{2}$ in $\left(G-x_{1}\right)^{\tau}$. In fact, $\tau$ contains at most one vertex from each connected component of $(G-I)^{\tau}$, since $x_{2}$ is adjacent to each $x \in \tau$ in the switch $\left(G-x_{1}\right)^{\tau}$. Notice that these connected components are trees, because $\left(G-x_{1}\right)^{\tau}$ is acyclic.

We extend the domain of $\tau$ by setting $\tau\left(x_{1}\right)=0$. Let $\tau=\left\{z_{1}, \ldots, z_{r}\right\}$. In Figure 5.1 we have depicted the graph

$$
G^{\tau}=\left(H+\left(T_{1} \cup T_{2} \cup \ldots \cup T_{r}\right)\right) \cup F,
$$

where 
- $H=K_{2, r}$ has the bipartition $\left(\left\{x_{1}, x_{2}\right\},\left\{z_{1}, \ldots, z_{r}\right\}\right)$,

- the induced subgraphs $T_{i}$ are disjoint trees with $H \cap T_{i}=\left\{z_{i}\right\}$, and

- $F$ is an acyclic induced subgraph or it is empty.

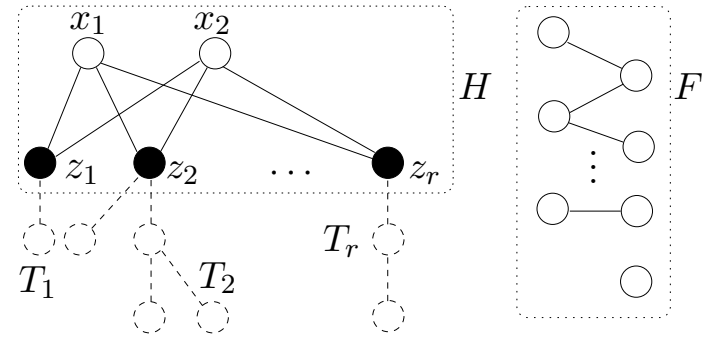

FIG. 5.1. The graph $G^{\tau}=\left(H+\left(T_{1} \cup T_{2} \cup \ldots \cup T_{r}\right)\right) \cup F$

In the following we let $C^{(i, j)}$ denote the cycle $\left(x_{1}, z_{i}, x_{2}, z_{j}\right)$ for different $i$ and $j$. Since $G^{\tau}$ is not acyclic, we must have $r \geq 2$ and thus $C^{(1,2)}$ always exists in $G^{\tau}$.

Claim 1. We have $|F| \leq 2$. Hence $F$ is either empty, discrete, or a path $P_{2}$. To see this, suppose that $F \neq \emptyset$. To avoid (7-1) and (7-2') in $C^{(1,2)} \cup F, F$ must be either discrete or a path $P_{2}$. If $F$ is discrete, then $|F| \leq 2$ by Lemma 5.1.

Claim 2. At most two of the trees $T_{1}, \ldots, T_{r}$ have more than one vertex.

Indeed, $x_{1}, x_{2}$ together with different $z_{i}, z_{j}, z_{k} \in \tau$ induce a $K_{2,3}$ in $G^{\tau}$. The graph (8-6) then implies the present claim.

Claim 3. Each nonsingleton tree $T_{i}$ has the form

$$
T_{i}=S_{k_{i}, s_{i}, 0} \quad \text { or } \quad T_{i}=P_{4}\left(s_{i}, 0\right),
$$

where $k_{i} \geq 0, s_{i} \leq 2$ and $z_{i}$ is the centre of $S_{k_{i}, s_{i}, 0}$ or it is the centre of $P_{4}\left(s_{i}, 0\right)$ adjacent to the $s_{i}$ leaves.

For this, let $T_{i}$ be a nonsingleton tree. By considering the subgraph of $G^{\tau}$ induced by $C^{(i, j)}$ and $T_{i}$ (for any $j \neq i$ ) we deduce that

- to avoid (7-1) the longest path of $T_{i}$ starting from $z_{i}$ has length at most 3 ,

- to avoid (7-1) each $v \neq z_{i}$ in $T_{i}$ with $v \notin N_{T_{i}}\left(z_{i}\right)$ satisfies $d_{T_{i}}(v) \leq 2$,

- to avoid (7-2) each $v \in N_{T_{i}}\left(z_{i}\right)$ satisfies $d_{T_{i}}(v) \leq 2$,

- to avoid (7-2') $T_{i}$ cannot have edge disjoint paths $P_{4}$ and $P_{3}$ with the common end $z_{i}$.

Hence $T_{i}$ has the required form. By Lemma 5.2, we have $s_{i} \leq 2$.

We shall divide our considerations according to the number $N$ of nonsingleton trees $T_{i}$.

Case $N=0$. In this case $G^{\tau}$ equals $K_{2, r} \cup F$ where $|F| \leq 2$. All these graphs have an acyclic switch. Indeed, in $K_{2, r}$ and $K_{2, r} \cup K_{1}$ we can take the selector $\left\{x_{1}, x_{2}\right\}$, in $K_{2, r} \cup 2 \cdot K_{1}$ and $K_{2, r} \cup P_{2}$ we can take $\left\{x_{1}, x_{2}, v\right\}$ where $v \in F$. These contradict the assumption that $G$ has no acyclic switches.

Case $N=1$. Suppose $G^{\tau}$ has a unique nonsingleton tree, say $T_{1}$, among the trees $T_{1}, \ldots, T_{r}$.

(1) Suppose first that $T_{1}=P_{4}\left(s_{1}, 0\right)$. We have $r=2$, for if $r \geq 3$, then $C^{(2,3)}$ together with $T_{1}-z_{1}$ does not avoid (7-1). Also, necessarily $F=\emptyset$ to avoid the graph $\left(7-2^{\prime}\right)$ in the subgraph induced by $C^{(1,2)}$ and $F$ together with the edge at the leaf of $T_{1}$. However, now $n \leq 9$ contradicts our assumption on the order $n$. 
(2) Suppose then that $T_{1}=S_{k_{1}, s_{1}, 0}$ with $k_{1}>0$.

(2.1) Assume that $r \geq 3$. Now $F=\emptyset, s_{1}=0$ and $k_{1}=1$, for otherwise the graph $\left(7-2^{\prime}\right)$ is in the subgraph induced by $C^{(2,3)}, F$ and $T_{1}-z_{1}$. Hence $T_{1}$ is a path $P_{3}$. However, now $G^{\tau}$ has an acyclic switch for all $r \geq 3$ (select all $z_{i}$ 's and the other end of the path $T_{1}$ ); a contradiction.

(2.2) Let $r=2$. To avoid (7-2'), $F$ cannot be $P_{2}$, and hence it is discrete or empty. However, now $G^{\tau}$ has an acyclic switch (select $z_{1}$ ); a contradiction.

(3) Finally, suppose that $T_{1}=S_{0, s_{1}, 0}$. Since $1 \leq s_{1} \leq 2$ and $|F| \leq 2$, we must have $r \geq 4$. By considering the graph induced by $C^{(2,3)}, F$ and $T_{1}-z_{1}$, we notice that to avoid (7-2') the subgraph $F$ cannot be $P_{2}$. To avoid (8-5) $\left(K_{\left\{x_{1}, x_{2}\right\},\left\{z_{1}, z_{2}, z_{3}\right\}}\right.$ with a leaf of $T_{1}$ and $F$ ) necessarily $|F| \leq 1$. If $|F|=1$, then $s_{1}=1$ to avoid the graph (8-5'). Hence $T_{1}$ is a path $P_{2}$. In both of these cases $\left(s_{1}=1\right.$ and $|F|=1$, and $s_{1}=2$ and $F=\emptyset$ ), we have an acyclic switch (select $x_{1}, x_{2}$ and a leaf adjacent to $z_{1}$ ); a contradiction.

Case $N=2$. Suppose that $G^{\tau}$ has exactly two nonsingleton trees, say $T_{1}$ and $T_{2}$, among the trees $T_{1}, \ldots, T_{r}$. Assume also without loss of generality that $\left|T_{1}\right| \geq\left|T_{2}\right|$.

To avoid (8-4) and (8-8) in the subgraph induced by $C^{(1,2)}$ and $F$, necessarily $|F| \leq 1$. Since $\left\{x_{1}, x_{2}, z_{1}, \ldots, z_{4}\right\}$ forms a $K_{2,4}$, we must have $r \leq 3$ in order to avoid (8-8'). Moreover, if $r=3$, then $F=\emptyset$ to avoid (8-7). In any case we have $r+|F| \leq 3$. Since $n \geq 10$, it follows that $\left|T_{1}\right|+\left|T_{2}\right| \geq(10-2)-(r-2)-|F| \geq 7$.

Let $t$ be the length of the longest path in $T_{1}$ starting from $z_{1}$. By Claim 3 we know that $t \leq 3$. First suppose that $t=1$ and hence that $T_{1}=S_{0, s_{1}, 0}$. We know $s_{1} \leq 2$ and hence $\left|T_{1}\right| \leq 3$ and we have $\left|T_{2}\right| \geq 4$, which contradicts the assumption $\left|T_{1}\right| \geq\left|T_{2}\right|$. Therefore $t \geq 2$. To avoid (8-1) in the subgraph induced by $C^{(1,2)}, T_{1}$ and $T_{2}$, necessarily $d_{T_{2}}\left(z_{2}\right)=1$. Moreover, to avoid (8-2), $T_{2}$ must be a path $P_{2}$. Consequently $\left|T_{1}\right| \geq 5$. If $T_{1}=S_{k_{1}, s_{1}, 0}$, then $k_{1}=1$. Indeed, if $k_{1} \geq 2$, we remove the middle vertex from one of the paths $P_{3}$ in $T_{1}$, to obtain the graph (8-3) in $G^{\tau}$. Now in all cases $\left|T_{1}\right|=t+1+s_{1} \geq 5$. However, the case where $t \geq 2$ and $s_{1}=2$ is excluded by (9-1), and the cases $t=3$ with $1 \leq s_{1} \leq 2$ are excluded by (8-4) (remove the neighbour of $z_{1}$ on the path longest path of $T_{1}$ starting from $z_{1}$ ).

Analogously to Lemma 5.2, we obtain

Lemma 5.5. Let $G$ be a critically cyclic graph of order $n \geq 10$. Then no vertex $v \in V$ is adjacent to more than one leaf of $G$.

We consider next isolated vertices in the subgraphs $G-x$ for critically cyclic graphs $G$.

LEMma 5.6. Let $G$ be a critically cyclic graph of order $n \geq 10$ and let $x \in V$.

(i) $G-x$ can have at most two isolated vertices. Moreover, if $G-x$ has two isolated vertices, then $x$ is adjacent to exactly one of these in $G$.

(ii) If a vertex $v \neq x$ is adjacent to $m$ leaves of $G-x$, then $m \leq 2$. Moreover, if $m=2$, then $x$ is adjacent to exactly one of these.

Proof. For (i) we only need to observe that if $G-x$ has three isolated vertices, then in either $G^{x}$ or $G$ at least two of these are isolated and we can apply Lemma 5.4. The same holds if the number of isolated vertices is two, but $x$ is not adjacent to exactly one of them in $G$.

For (ii) assume that there is a vertex $v \neq x$ adjacent to more than two leaves. The vertex $x$ is nonadjacent to at least two of these in either $G$ or $G^{x}$ and the result then follows from Lemma 5.5.

Let $G$ be a graph and $x$ and $y$ be vertices of $G$ such that $G-x$ is acyclic. We say that $y$ is compatible with $x$ if $G-y$ and $G^{x}-y$ are not acyclic. 
Note that if $y$ is compatible with $x$, then all cycles in $G$ (and $G^{x}$ ) go through $x$, and some but not all of them go through $y$.

LEMmA 5.7. Let $G$ be critically cyclic graph such that $G-x$ is acyclic.

(i) If $y$ is compatible with $x$, then $G-\{x, y\}$ is a special acyclic graph.

(ii) If $G$ is of order $n \geq 8$ such that $G \notin\left[C_{n}\right]$, then there exists a vertex $y \in V$ that is compatible with $x$.

Proof. Since $G$ is critically cyclic, $G-y$ has an acyclic switch $(G-y)^{\tau}$. Let $S=G-\{x, y\}$. Because $S$ and $S^{\tau}$ are both acyclic graphs it follows that either $S$ is special or $\tau$ is constant on $S$.

Suppose $\tau$ is constant on $S$, and thus either $\tau=S \cup\{x\}$ or $\tau=S$. In the former case $(G-y)^{\tau}=G-y$ is a contradiction, because $(G-y)^{\tau}$ is acyclic and $G-y$ is not. In the second case we have $(G-y)^{\tau}=\left(G^{x}-y\right)^{S \cup\{x\}}=G^{x}-y$. Again we have a contradiction, since $G^{x}-y$ is supposed to have a cycle. This proves that $S$ is special.

For (ii), suppose $G \notin\left[C_{n}\right]$. Since $G$ has no acyclic switches, there are cycles in $G$ and $G^{x}$, and they all pass through $x$, because $G-x$ is acyclic. Moreover, since $C_{k}$ is critically cyclic for $k \geq 7$, the induced cycles of $G$ and $G^{x}$ have length at most 6 .

If $G$ or $G^{x}$ has an induced cycle $C_{5}$ or $C_{6}$, let $y$ be a vertex that is not on such a cycle. It is clear that $G-y$ and $G^{x}-y$ both contain a cycle, and therefore each such vertex $y$ is compatible with $x$.

If $G$ and $G^{x}$ have both an induced cycle of length at most 4, then these two cycles have altogether at most 7 vertices (since they share the vertex $x$ ). Since $n \geq 8$, there exists a vertex $y$ that is not on these cycles. For such a vertex $y$, both $G-y$ and $G^{x}-y$ are not acyclic. This proves the claim.

The next lemma is our second main tool for isolated vertices.

LEMma 5.8. Let $G$ be a critically cyclic graph of order $n \geq 10$ such that $G-x$ is acyclic. Then $G-x$ has no isolated vertices.

Proof. Assume to the contrary of the claim that there is an isolated vertex $u$ in $G-x$. Now $u$ is either a leaf adjacent to $x$ in $G$ (and hence isolated in $G^{x}$ ) or it is isolated in $G$ (and hence a leaf adjacent to $x$ in $G^{x}$ ). Hence no cycle goes through $u$ in $G$ or $G^{x}$. It follows that $G-u$ and $G^{x}-u$ are not acyclic and by Lemma 5.7(i), $S=G-\{x, u\}$ is special.

Let $\tau$ be a selector for which $(G-u)^{\tau}$ is acyclic. Since $G-u$ and $G^{x}-u$ both have a cycle, $\tau$ is not constant on $S$.

If $S=S_{A}(k, m)$ is of the type (As) for $A \in\{2, \ldots, 8\}$, then by Lemma 5.6(ii), the centres of $S$ are adjacent to at most two leaves of $G-x$ and hence $k, m \leq 2$. In this case, $S$ has at least eight vertices (since $n \geq 10)$ and this rules out the types $(2 \mathrm{~s}$ ), $(3 \mathrm{~s}),(4 \mathrm{~s})$ and $(6 \mathrm{~s})$. Thus $S$ is of the type (1s) or it is one of the types $(5 \mathrm{~s}),(7 \mathrm{~s}),(8 \mathrm{~s})$ with $k=2=m$.

In the cases $(5 \mathrm{~s}),(7 \mathrm{~s})$ and $(8 \mathrm{~s})$, by Lemma $4.3, S$ has a singular acyclic switch with respect to its centres $Z(S)=\left\{z_{1}, z_{2}\right\}$ and therefore $\tau=\left\{z_{1}, z_{2}\right\}$ or $\tau=\left\{x, z_{1}, z_{2}\right\}$. However, by Lemma 5.6(ii), $x$ is adjacent to two leafs of $S$, one leaf being adjacent to $z_{1}$ and one to $z_{2}$. The same holds for $S^{\tau}$.

This means that $(G-u)^{\tau}$ is not acyclic, which is a contradiction.

Consider then the case $S=S_{k, m, l}$ and adopt the notations (S1) - (S3) for it. Without restriction we can assume that $\tau(z)=1$ for the centre $z$ of $S$, since $(G-u)^{\bar{\tau}}=$ $(G-u)^{\tau}$. Extend $\tau$ to the whole domain by setting $\tau(u)=0$.

We have $n=(2 k+1)+m+l+2 \geq 10$, and thus $k \geq \frac{1}{2}(7-(m+l))$. By Lemma 5.6, $m \leq 2$ and $l \leq 1$. (Recall that $u$ is isolated in $G-x$.) In particular, $k \geq 2$, and if $k=2$, then $m=2, l=1$ and $n=10$. (In Figure 5.2 we have depicted the graph 
$S_{k, m, l}$ obtained so far. In solid lines we indicate what must be there, in dotted lines what may be there.)

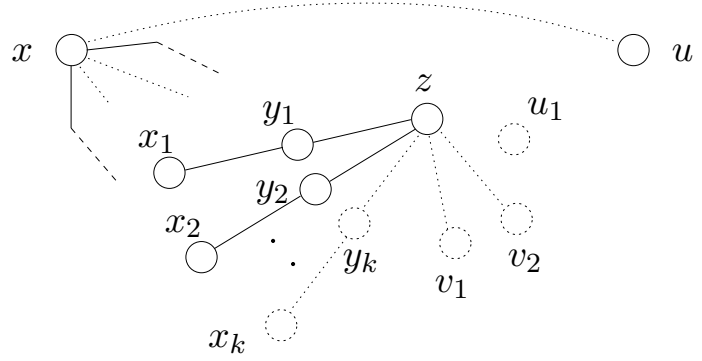

FIG. 5.2. The case of the special graph $S_{k, m, l}$

In these cases, by Lemma 4.4, the special acyclic graph $S$ has a singular acyclic switch $S^{\rho}$ for $\rho=\{z\}$. Now $(G-u)^{\tau}$ is acyclic and hence so is $S^{\tau}$. Since $\tau$ is not constant on $S$, the uniqueness of $\rho$ implies that $\rho(v)=\tau(v)$ for all $v \notin\{x, u\}$. Also, the only vertices in $G$ that can be adjacent to $u$ in $G^{\tau}$ are $x$ and $z$ and because $G^{\tau}$ is not acyclic, both must be adjacent to $u$. Moreover, $x$ is adjacent in $G^{\tau}$ to exactly one vertex $v \in H \cup I$, since $G^{\tau}$ has a cycle but $G^{\tau}-u\left(=(G-u)^{\tau}\right)$ is acyclic.

Suppose that $v$ is a leaf of the part $H$ of $S$, say $v=x_{1}$. If $l \geq 1$, then $\left\{x, x_{1}, z, u, y_{1}, u_{1}, y_{2}\right\}$ induces a graph (7-4) in $G^{\tau}$. Similarly, if $m \geq 1$, then $\left\{x, x_{1}, z, u, y_{1}, w, v_{1}\right\}$ induces a graph (7-4) in $G^{\tau}$ where $w=x_{2}$ if $G^{\tau}\left(x v_{1}\right)=1$ and $w=y_{2}$ otherwise. Therefore we have $m=0=l$. Now $k \geq 4$, and $G^{\tau}$ contains an induced graph (7-4) obtained by removing $x_{2}$.

If $v$ is a middle vertex in $H$, say $v=y_{1}$, then $\left\{x, y_{1}, x_{1}, z, u\right\}$ induces a cycle $C_{5}$ in $G^{\tau}$, and hence $G^{\tau}$ has an induced graph (6-1') obtained by removing $x_{2}$.

If $v \in I$ is isolated in $S$, say $v=u_{1}$, then to avoid (8-3) as being induced by the set $\left\{x, u_{1}, z, u, x_{1}, y_{1}, y_{2}, v_{i}\right\}$ (for any $v_{i} \in M$ ), we must have $G^{\tau}\left(x v_{i}\right)=0$ (provided that $m>0)$. However, now $\left(G^{\tau}\right)^{z}$ is acyclic; a contradiction.

Finally, if $v=z$, then again $\left(G^{\tau}\right)^{z}$ is acyclic. This contradiction completes the proof of the lemma.

6. The proof of Theorem 3.5. In the following we shall consider every type of special acyclic graphs in turn and show that each case leads to a contradiction, thereby proving our main theorem that besides the graphs in $\left[C_{n}\right]$ there are no critically cyclic graphs of order $n \geq 10$.

Throughout this section we let $G$ be a critically cyclic graph of order $n=|V| \geq 10$ such that $G \notin\left[C_{n}\right]$. Also, let $x \in V$ be a fixed vertex.

Since $G$ is critically cyclic, there exists an acyclic switch $(G-x)^{\rho}$ of the subgraph $G-x$. Since the switches of critically cyclic graphs are critically cyclic, we can assume that $\rho$ is constant on $V$, and therefore that $G-x$ is acyclic already.

By Lemma 5.7(ii), there exists a vertex $y$ that is compatible with $x$, that is, $G-y$ and $G^{x}-y$ are not acyclic. Since $G$ is critically cyclic, there is a nonconstant selector $\sigma$ such that $(G-y)^{\sigma}$ is acyclic. By Lemma $5.7(\mathrm{i}), S=G-\{x, y\}$ is a special acyclic graph, and it is of one of the types $(1 \mathrm{~s})-(8 \mathrm{~s})$, since its order is at least 8 .

In the following proofs a number of simple properties are often used, and we note them here: first of all, the vertex $y$ is adjacent to at most one vertex of each component of $S$. If not, $G-x$ would not be acyclic. Also, there must be a cycle in $G$ that does not contain $y$, because $G-y$ is not acyclic. This also holds for $G^{x}-y$. 
We shall now formulate a few conditions that hold for the special acyclic graphs $(1 \mathrm{~s})-(8 \mathrm{~s})$. For any graph $G^{\prime}$ and a vertex $v$, let $L_{G^{\prime}}(v)$ be the set of leaves adjacent to $v$ in $G^{\prime}$, and let $I_{G^{\prime}}$ denote the set of isolated vertices in $G^{\prime}$.

LEMMA 6.1. In the above notations, we have

(i) $I_{S} \subseteq N_{G}(y)$.

(ii) for all $v \in S,\left|L_{S}(v)\right| \leq 3$. Moreover, $\left|L_{S}(v)\right|=3$ implies $\left|N_{G}(x) \cap L_{S}(v)\right| \geq 1$ and $\left|N_{G}(y) \cap L_{S}(v)\right|=1$.

Proof. By Lemma 5.8, $G-x$ does not have isolated vertices, and hence Claim (i) follows.

For (ii), we have $\left|N_{G}(y) \cap L_{S}(v)\right| \leq 1$, since $G-x$ is acyclic. If $\left|L_{S}(v)\right| \geq 3$, then, by Lemma $5.6(\mathrm{ii}),\left|L_{S}(v)-N_{G}(y)\right| \leq 2$, and $x$ is adjacent to at most one vertex of $L_{S}(v)-N_{G}(y)$. Hence, in this case, we must have $\left|L_{S}(v)\right|=3$ and in this case $x$ and $y$ are each adjacent to at least one vertex in $L_{S}(v)$. Because $G-x$ is acyclic, $y$ is adjacent to exactly one vertex in $L_{S}(v)$.

Note how the previous Lemma restricts the values of $k$ and $m$ for the types (2s)$(8 \mathrm{~s})$ and $m$ for $(1 \mathrm{~s})$. On the other hand $n \geq 10$ gives a lower bound on these values for most types.

6.1. The case (1s). We shall now consider first the most difficult case, $S=$ $S_{k, m, l}$. We adopt the notations of (S1) - (S3) for it. Without restriction we may assume that $\sigma(z)=1$ for the centre $z$ of $S$. Also, we can assume that $\sigma(x)=0$, by the symmetry in the definition of compatibility, i.e., by the fact that both $G-y$ and $G^{x}-y$ are not acyclic. We extend $\sigma$ to the whole domain by setting $\sigma(y)=0$. Note that $(G-y)^{\sigma}=G^{\sigma}-y$.

LEMma 6.2. We have

(i) $k=2$,

(ii) $1 \leq l \leq 2,1 \leq m \leq 2$ and $m+l \geq 3$,

(iii) $M \subseteq N_{G}(x)$,

(iv) if $l=2$, then $\left|N_{G}(x) \cap I\right|=1$,

(v) if $m=2$, then $\left|N_{G}(y) \cap M\right|=1$,

(vi) $\left|N_{G}(x) \cap(H \cup I)-\{z\}\right| \leq 1$.

Proof. By Lemma 5.4, $G$ has at most one isolated vertex, therefore $\left|N_{G}(x) \cap I\right| \leq$ 1 for otherwise, switching by $\{x, y\}$ we obtain two isolated vertices, because $y$ is connected to all vertices in $I$ by Lemma 6.1. When Lemma 5.6(ii) is applied to the vertex $y$, we have that $l \leq 2$ and also Claim (iv).

If $k=0$, then $m+l \geq 7$, since $n \geq 10$. This contradicts the bound $m \leq 3$ of Lemma 6.1(ii) and the above bound $l \leq 2$. Hence $k \geq 1$.

If $k=1$, then $m+l \geq 5$. In this case, $l=2$ and $m \geq 3$. If $k=2$, then $m+l=3$, using reasoning similar to that for $k=1$. By Lemma 4.4 in both cases $S^{z}$ is the singular acyclic switch of $S$ and thus $\sigma=\{z\}$. Now $M \subseteq N_{G}(x)$, for otherwise, there is an isolated vertex of $M$ in the acyclic graph $G^{\sigma}-y$, contradicting Lemma 5.8 (recall that $\sigma(x)=0=\sigma(y)$ ). Therefore Claim (iii) is true. Moreover, when Lemma 5.6(ii) is applied to $G^{\sigma}$, it follows that $m \leq 2$, and as a consequence $k \geq 2$, because as it was shown above if $k=1$, then we must have $m=3$. The Claim (v) follows now from Lemma 5.6(ii).

Claim (vi) follows from the fact that the subgraph of $G^{\sigma}$ induced by $H \cup I$ is connected and $G^{\sigma}-y$ is acyclic.

Suppose then that $k \geq 3$. By Claim (vi) it follows that there are at least two pairs $x_{i} y_{i}$ such that $G\left(x x_{i}\right)=0=G\left(x y_{i}\right)$, say for $i=1,2$. For $i=1,2$ let $\tau_{i}$ be such that $\left(G-x_{i}\right)^{\tau_{i}}$ is acyclic, where we may choose $\tau_{i}(z)=1$. The special 
acyclic graph $S-x_{i}$, which is $S_{k-1, m+1, l}$, has a singular acyclic switch $\left(S-x_{i}\right)^{z}$ : $n=2 k+m+l+|\{x, y, z\}| \geq 10$ and $k \geq 3$ implies $m+l \geq 1$, so that in the case of $S_{k-1, m+1, l}$ we may apply Lemma 4.4).

Clearly $\tau_{i}=\sigma$ when we set $\tau_{i}\left(x_{i}\right)=0$. By Lemma 5.8, the vertex $y_{i}$ is not isolated in $G^{\tau_{i}}-x_{i}$, and therefore $G^{\tau_{i}}\left(y y_{i}\right)=1=G\left(y y_{i}\right)$ for $\mathrm{i}=1,2$ (since $G\left(x y_{i}\right)=$ $\left.0=G^{\tau_{i}}\left(x y_{i}\right)\right)$ and we have a cycle in $G-x$. This contradiction proves Claim (i) and Claim (ii).

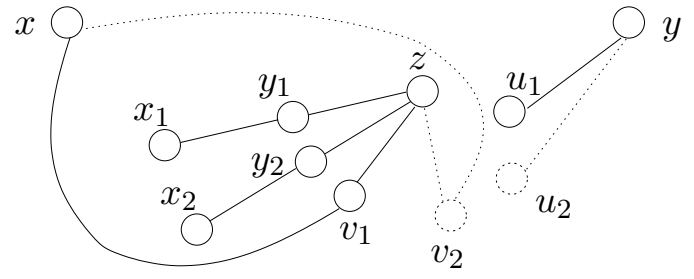

FIG. 6.1. The case of the special graph $S_{k, m, l}$ after Lemma 6.2

In Figure 6.1 we have depicted part of the situation for the special graph $S_{k, m, l}$ : the demands $I_{S} \subseteq N_{G}(y), k=2,1 \leq m \geq 2,1 \leq l \leq 2, M \subseteq N_{G}(x)$ are all implied by the drawing (again solid means what must be there, dotted means what might be there). Notice that in the case at hand, Lemma 6.2 implies that $n \leq 11$.

We shall now finish the case $S=S_{k, m, l}$. In the following we consider the adjacencies of $x$ and $y$ to vertices in $S$ and each other.

(1) Assume first that $x$ is adjacent to a vertex $u$ in $I$. By the cases (ii) and (iv) of Lemma $6.2, u$ is the only neighbour of $x$ in $I$. Then also $G(x z)=1$, since otherwise, $(x, u, z)$ is a triangle in $G^{\sigma}-y$, contradicting the fact that $G^{\sigma}-y$ is acyclic. Moreover, $G\left(x x_{i}\right)=0=G\left(x y_{i}\right)$ for $i=1,2$, because $G^{\sigma}-y$ is acyclic. We have $G(x y)=0$, for otherwise, $(x, y, u)$ is a triangle in $G$, and to avoid (5-1) with the edges $G\left(x_{i} y_{i}\right)=1$, we would have to have that $y$ is adjacent to two vertices in $H-\{z\}$ giving a cycle to $G-x$. Now we know that $N_{G}(x)=M \cup\{z, u\}$, by Lemma 6.2(iii).

For any vertex $v \in M,(x, z, v)$ is a triangle in $G$. Consider the subgraph of $G$ induced by $\left\{x, u, y, v, z, x_{1}, y_{1}\right\}$. To avoid (7-5') we must have that either $G(y z)=1$ or $G(y v)=1$. Since $G^{\sigma}-y$ is acyclic, $y$ is adjacent in $G$ to no other vertices of $H \cup M \cup\{x\}$.

(1.1) Suppose $G(y z)=1$. By Lemma $6.2(\mathrm{v})$, we have $m=1$ and so $M=\{v\}$. Then Lemma 6.2(ii) implies that $l=2$. But now $\left\{x, u_{1}, y, z, y_{1}, x_{2}, u_{2}\right\}$ induces the graph (7-4) in $G$; a contradiction.

(1.2) Suppose $G(y v)=1$. Then $\left\{u_{1}, y, x, v_{1}, z, y_{1}, x_{2}\right\}$ induces a (7-3); also a contradiction.

(2) Therefore $G(x u)=0$ for all $u \in I$ and thus by Lemma 6.2(iv), $l=1, I=\left\{u_{1}\right\}$. We have from the case (ii) of Lemma 6.2 that $m=2$ (and hence $M=\left\{v_{1}, v_{2}\right\}$ ) and from the case (v) that $y$ is adjacent to exactly one vertex of $M$, say $G\left(y v_{1}\right)=1$. In conclusion so far we know that $G\left(x v_{1}\right)=1=G\left(x v_{2}\right), G\left(y v_{1}\right)=1, G\left(y v_{2}\right)=0$, and $G\left(y u_{1}\right)=1, G\left(x u_{1}\right)=0$. Also, $G(y w)=0$ for all $w \in S-\left\{u_{1}, v_{1}\right\}$, since $G-x$ is acyclic.

Since $G^{\sigma}-y$ is acyclic, we must have $G\left(x x_{i}\right)=0=G\left(x y_{i}\right)$ for $i=1$ or 2 , say $i=1$. There are two cases to be considered here.

(2.1) Suppose $G(x z)=0$. Now $G(x y)=1$, since otherwise, $\left\{x, v_{1}, v_{2}, z, y, x_{1}, y_{1}, u_{1}\right\}$ induces a graph (8-9) in $G$. 
(2.2) Suppose $G(x z)=1$. To avoid $\left\{x, z, v_{1}, y_{1}, x_{1}, y, u_{1}\right\}$ inducing a (7-5') we must have $G(x y)=1$.

In both of these cases, we have $G(x y)=1$. But now $\left\{x, y, v_{1}, x_{1}, y_{1}\right\}$ induces a graph (5-1'). This contradiction proves that the special graph $S=G-\{x, y\}$ is not of the type (1s).

6.2. The other cases. Let $S=S_{A}(k, m)$ for $A \in\{2, \ldots, 8\}$ where we assume that $k \geq m$. Let $z_{1}$ and $z_{2}$ be the two centres of $S$, and $L=\left\{v_{1}, v_{2}, \ldots, v_{k}\right\}$ and $M=\left\{u_{1}, u_{2}, \ldots, u_{m}\right\}$ be the sets of leaves of $S$ adjacent to $z_{1}$ and $z_{2}$, respectively.

We may assume that $\sigma\left(z_{1}\right)=1$ and, as in the previous case, we can assume that $\sigma(x)=0$. Again we extend $\sigma$ to the whole domain by setting $\sigma(y)=0$.

Lemma 6.3. We have $k \leq 3$. Moreover,

(i) if $S$ is of the types (3s) - (8s), then $m \leq 2$.

(ii) if $k=3$ and if $S^{\sigma}$ is the singular acyclic switch of $S$, then $x$ and $y$ are each adjacent to exactly one, but different vertex in $L$.

Proof. The fact $k \leq 3$ is already stated in Lemma 6.1(ii).

For (i), assume that $m \geq 3$, and thus that both $z_{1}$ and $z_{2}$ have three leaves adjacent to them in $S$. By Lemma 6.1(ii), $y$ is adjacent to a leaf in both $L$ and $M$. For the types (4s) - (8s) (where $S$ is connected) $G-x$ has a cycle; a contradiction. For the type (3s) we apply the same argument but taking $y$ in $G^{\sigma}$ ) instead of $x$ : we first observe that $S^{Z(S)}$ has a singular acyclic switch by Lemma 4.3, and therefore $\sigma=Z(S)$. Now $x$ is adjacent in $G^{\sigma}-y$ to a leaf in both $L$ and $M$. It follows then that $G^{\sigma}-y$ has a cycle; a contradiction.

For the claim (ii), we first observe that $x$ is adjacent to exactly one vertex in $L$, since $G^{\sigma}-y$ is acyclic. The claim then follows from Lemma 5.6(ii) and Lemma 6.1(ii).

Note that by Lemma 6.1(ii), Lemma 6.2(i) and (ii), Lemma 6.3(i) it already follows that there are no critically cyclic graphs of order at least 12 (unless they are in $\left.\left[C_{n}\right]\right)$.

6.3. The cases (2s)-(4s). Let $S$ be of the type (2s), (3s) or (4s). Since $n \geq 10$, Lemma 6.1(ii) implies that $k=3$ and $2 \leq m \leq 3$. By Lemma 4.3, $S$ has a singular acyclic switch which means that $\sigma=\left\{z_{1}, z_{2}\right\}$. (Recall that $\sigma\left(z_{1}\right)=1$ and $\sigma(x)=0=$ $\sigma(y)$.)

By Lemma 6.3(ii), $x$ is adjacent to one vertex in $L$, say $G\left(x v_{1}\right)=1$, and $y$ is adjacent to another vertex of $L$, say $G\left(y v_{3}\right)=1$. Furthermore, $G\left(x v_{2}\right)=0=G\left(y v_{2}\right)$ for the third vertex $v_{2}$ of $L$. Since $G\left(z_{2} v_{1}\right)=0$ and $G\left(x v_{1}\right)=1$, we must have that $G\left(x z_{2}\right)=1$, for otherwise, there is a cycle in $G^{\sigma}-y$. We now run through the cases one by one.

Case (2s). Let $S=S_{2}(k, m)$. By Lemma 6.3 and the fact that $n \geq 10$, we know that $k=3=m$. Also by Lemma 6.3(ii), $x$ and $y$ are both adjacent to one but different vertex in $M$, say $G\left(x u_{1}\right)=1$ and $G\left(y u_{3}\right)=1$. By the uniqueness of $\sigma, x$ must be adjacent to $z_{1}$ to ensure that $G^{\sigma}-y$ is acyclic $\left(\left\{z_{1}, x, u_{1}\right\}\right.$ would be a triangle). Because $y$ can only be connected to one vertex in every component of $S$, the only remaining unknown is $G(x y)$. If $G(x y)=0$, then $\left\{x, v_{1}, z_{1}, u_{3}, y\right\}$ induced the graph $(5-1)$, and if $G(x y)=1$, then $\left\{u_{1}, x, y, v_{3}, z_{1}, v_{2}, u_{2}\right\}$ induces the graph (7-4). These contradictions show that $S$ is not of the type $(2 \mathrm{~s})$.

Case (3s). Let $S=S_{3}(k, m)$. In this case $S^{\sigma}$ is of the type (4s).

By the above, $G\left(x z_{2}\right)=1$ and $G\left(x v_{2}\right)=0=G\left(x v_{3}\right)$. To avoid a cycle in $G^{\sigma}-y$, necessarily $G\left(x z_{1}\right)=1$. $G(x w)=0$ (for the isolated vertex $w$ of $G$ ) and $G(x u)=0$ 
for all $u \in M$. The reason is that $x$ is adjacent to $v_{1}$ in $S^{\sigma}$, and the above choices prevent $x$ from being adjacent to any other vertex of the connected graph $S^{\sigma}$.

By Lemma 5.6(ii), $m=2$ and $y$ is adjacent to one vertex in $M$, say $G\left(y u_{2}\right)=1$. By Lemma 6.1(i), $y$ is connected to $w$. The only unknown is the value $G(x y)$. If $G(x y)=0$, then $\left\{v_{1}, x, z_{2}, u_{1}, y, v_{3}, z_{1}\right\}$ induces the graph (7-5'), and if $G(x y)=1$, then $\left\{v_{1}, x, y, u_{2}, z_{2}, v_{2}, u_{1}\right\}$ induces the graph (7-4). Hence $S$ is not of the type (3s).

Case (4s). Let $S=S_{4}(k, m)$. Because $S$ is connected, $y$ is not adjacent to any other vertex of $S$ (except $v_{3}$ ). Hence, $m=2$ and $x$ is adjacent to one vertex of $M$, say $G\left(x u_{1}\right)=1$ (see Lemma 5.6(ii)). Since $G^{\sigma}-y$ is acyclic, $x$ must be adjacent to $z_{1}$. If $G(x y)=0$, then $\left\{x, u_{1}, z_{2}, v_{3}, y\right\}$ induces the graph (5-1). If $G(x y)=1$, then $\left\{v_{1}, z_{1}, v_{2}, v_{3}, x, u_{1}, y\right\}$ induces the graph (7-4) in $G^{x}$. Hence $S$ is not of the type (4s).

6.4. The cases $(5 \mathrm{~s})-(8 \mathrm{~s})$. We shall first consider the type (6s).

Case (6s). Let $S=S_{6}(k, m)$. In this case, $n \geq 10$ implies that $k, m \geq 3$. But this contradicts Lemma 6.3(i). Hence $S$ is not of the type (6s).

For the remaining cases $(5 \mathrm{~s}),(7 \mathrm{~s})$ and $(8 \mathrm{~s})$, let $w_{1}$ be the neighbour of $z_{1}$ of degree at least 2. Let $w_{2}$ be the vertex that is not adjacent to $z_{1}$ and $z_{2}$ in the types (5s) and $(7 \mathrm{~s})$, and which is adjacent to $z_{2}$ in $(8 \mathrm{~s})$ (see Figure $4.1(5 \mathrm{~s}),(7 \mathrm{~s})$ and $(8 \mathrm{~s})$ ).

By Lemma 6.1(ii) and $n \geq 10,2 \leq k \leq 3, m \geq 1$, and $k+m \geq 4$. In all these cases $S$ has a singular acyclic switch by Lemma 4.3 and therefore $\sigma=\left\{z_{1}, z_{2}\right\}$.

We can assume that $x$ is adjacent to a vertex in $L$, say $G\left(x v_{1}\right)=1$. This follows from Lemma 6.3(ii) if $k=3$. On the other hand, if $k=2$, then necessarily $m=2$, since $k+m \geq 4$, and in this case $N_{G}(y) \cap L=\emptyset$ or $N_{G}(y) \cap M=\emptyset$ in order to avoid a cycle in $G-x$. By Lemma 5.6(ii), $N_{G}(x) \cap M \neq \emptyset$ or $N_{G}(x) \cap L \neq \emptyset$, respectively. In the case under consideration, $k=m(=2)$, so the assumption that $G\left(x v_{1}\right)=1$ is validated.

Claim 1: The following adjacencies for $x$ exist: $G\left(x z_{1}\right)=1=G\left(x z_{2}\right)$, and $G(x u)=0$ for all $u \notin\left\{v_{1}, z_{1}, z_{2}, w_{2}, y\right\}$. Moreover, $G\left(x w_{2}\right)=0$ if $d_{S}\left(w_{2}\right) \neq 0$ (that is, excepting the case $(5 \mathrm{~s}))$.

Recall that $\sigma=\left\{z_{1}, z_{2}\right\}$ (and $\sigma(x)=0$ ). In the cases under consideration the centres $z_{1}$ and $z_{2}$ belong to the same connected component as $v_{1}$ in both of the acyclic graphs $S$ and $S^{\sigma}$. Since $G^{\sigma}-y$ is acyclic, $x$ can be adjacent in $G^{\sigma}-y$ only to $v_{1}$. Hence $G\left(x z_{1}\right)=1=G\left(x z_{2}\right)$. Also, if $d_{S}\left(w_{2}\right)>0$, then $w_{2}$ is in the same connected component, from which it follows that $G\left(x w_{2}\right)=0$ as required.

Claim 2: The following adjacencies for $y$ exist: $G(y v)=1$ holds for exactly one vertex $v \in S-\left\{w_{2}\right\}$, and either

(i) $v \in L$, say $G\left(y v_{3}\right)=1$, in which case $k=3$ and $m=1$,

(ii) $v \in M$, say $G\left(y u_{2}\right)=1$, in which case $k=2, m=2$.

Moreover, $G\left(y w_{2}\right)=1$ holds only in the case $(5 \mathrm{~s})$.

For the first statement we observe that $S$ is connected in the cases $(7 \mathrm{~s})$ and $(8 \mathrm{~s})$ and $S-w_{2}$ is connected in the case $(5 \mathrm{~s})$. Hence $y$ is adjacent to at most one vertex in $S-w_{2}$, since $G-x$ is acyclic. By Lemma $5.8, G-x$ does not have isolated vertices and therefore $G(y v)=1$ for a unique $v \in S-\left\{w_{2}\right\}$ as required.

Now if $y$ is not adjacent to a vertex of $M$, then $|M|=1$ by Lemma 5.6(ii) and the fact that $G(x u)=0$ for all $u \in M$. It follows that $k=3$, and, consequently, $y$ is adjacent to a vertex of $L$. On the other hand, if $G(y u)=1$ for a $u \in M$, then $G(y v)=0$ for all $v \in L$ to avoid a cycle in $G-x$, and in this case, $k=2$ by Lemma 5.6. 
That $G\left(y w_{2}\right)=1$ in the case (5s) follows from Lemma 6.1(i). In the other two cases, $G\left(y w_{2}\right)=1$ would result in a cycle in $G-x$.

These two claims together determine $G$ with the exception of the value for $G(x y)$. We are ready to exclude the remaining cases.

Case (5s). Let $S=S_{5}(k, m)$. Now $x$ is not adjacent to $w_{1}$ and neither is $y$. Hence in $G^{\sigma}-y$ the vertex $w_{1}$ is isolated, which contradicts Lemma 5.8.

Case (7s). Let $S=S_{7}(k, m)$. In both cases (i) and (ii) of Claim 2, $G(x y)=1$ to avoid (7-4) as being the subgraph induced by the vertices $\left\{x, z_{1}, w_{1}, z_{2}, v_{2}, w_{2}, y\right\}$. Now $G$ contains a switch of the graph (7-4) if $k=3$ and $m=1$ (this is $G^{\left\{z_{1}\right\}}-\left\{w_{1}, w_{2}, u_{1}\right\}$ ), and $G$ contains the graph (7-5') if $k=2=m$ (this is $G-\left\{u_{1}, v_{2}, z_{2}\right\}$ ).

Case (8s). Let $S=S_{8}(k, m)$. In both cases (of Claim 2), $G(x y)=1$ to avoid (6-1) as being the subgraph induced by the vertices $\left\{x, z_{1}, w_{1}, w_{2}, z_{2}, y\right\}$. Now $\left\{x, z_{1}, w_{1}, w_{2}, z_{2}, y, u_{1}\right\}$ induces the graph (7-3').

This proves Theorem 3.5 .

7. Concluding remarks. Finding the critically cyclic graphs was done as follows: a program was written in $\mathrm{C}$ that listed for a number $n$ of vertices, a representative of each switching class that did not contain any acyclic switches. In a later phase, when we were looking for critically cyclic graphs on $n$ vertices, we only had to make sure that all critically cyclic graphs of lower order could not anymore occur in these graphs. The program was run in this way for up to 12 vertices. We used here the files from [10] which list generators for the switching classes up to isomorphism and up to complementation for up to 10 vertices.

A computer program in the functional language Scheme verified that the critically cyclic graphs found were in fact critically cyclic. Also, the authors verified this by hand.

In our proofs, not all of the critically cyclic graphs were used. The graphs that were not used are (8-10)-(8-15) and (9-3)-(9-5). Lemma 5.7 excludes the cycles $C_{8}$ and $C_{9}$. For the other graphs, except (8-12), the reason is that if they are induced subgraphs of any graph of order at least 10, then this graph also contains one of the cyclic graphs from Figure 3.1, 3.2 and 3.3 or it contains (8-12). The graph (8-12) does not occur in our proofs, because it is overruled by Lemmas 5.7 and 5.8, that is, if $G$ is a forbidden graph of order 10 that does not have 2 isolated vertices and such that $G-x$ is acyclic and $G-\{x, y\}$ is special, then $G$ contains an induced critically cyclic graph that was used in the proofs.

As an aside we note that our program found that the graphs (8-9) and (8-12) have a similar property: adding two vertices to either of these graphs in any way, always results in a graph that contains a switch of one of the other critically cyclic graphs.

\section{REFERENCES}

[1] D. ACHARYA, On characterizing graphs switching equivalent to acyclic graphs, Indian J. Pure Appl. Math, 12 (1981), pp. 1187-1191.

[2] P. Cameron, Cohomological aspects of two-graphs, Math. Z., 157 (1977), pp. $101-119$.

[3] D. Corneil and R. Mathon, eds., Geometry and Combinatorics: Selected Works of J.J. Seidel, Academic Press, Boston, 1991.

[4] A. Ehrenfeucht, T. Harju, and G. Rozenberg, The Theory of 2-Structures, World Scientific, 1999.

[5] J. HAge, Structural Aspects Of Switching Classes, PhD thesis, LIACS, 2001. http://www.cs.uu.nl/people/jur/2s.html. 
[6] J. Hage and T. HarJu, Acyclicity of switching classes, European J. Combin., 19 (1998), pp. 321-327.

[7] J. v. Lint and J. Seidel, Equilateral points in elliptic geometry, in Proc. Kon. Nederl. Acad. Wetensch., Ser. A, vol. 69, 1966, pp. 335 - 348. Reprinted in [3].

[8] J. Seidel, A survey of two-graphs, in Colloquio Internazionale sulle Teorie Combinatorie (Rome,1973), vol. I, Rome, 1976, Acc. Naz. Lincei, pp. 481-511. Reprinted in [3].

[9] J. SEIDEl AND D. TAYlor, Two-graphs, a second survey, in Algebraic Methods in Graph Theory (Proc. Internat. Colloq., Szeged, 1978), L. Lovasz and V. Sós, eds., vol. II, Amsterdam, 1981, North-Holland, pp. 689-711. Reprinted in [3].

[10] E. Spence, Tables of two-graphs. http://gauss.maths.gla.ac.uk/ ted/.

[11] T. Zaslavsky, A Mathematical Bibliography of Signed and Gain Graphs and Allied Areas, vol. DS8 of Dynamic Surveys in Combinatorics, World Combinatorics Exchange, http://www.combinatorics.org, 1999. 